\title{
EFEITO DO TEOR INICIAL DE ÁGUA DE SEMENTES DE FEIJÃO E DA CÂMARA NO TESTE DE ENVELHECIMENTO ACELERADO1
}

\author{
Angelo Scappa Neto ${ }^{2,4}$; Sonia Regina Mudrovitsch de Bittencourt²; Roberval Daiton Vieira ${ }^{2,5 *}$ \\ Clovis Alberto Volpe ${ }^{3}$ \\ ${ }^{2}$ Depto. de Produção Vegetal, FCAV/UNESP, Via de acesso Prof. Paulo Donato Castellane, s/n - CEP: 14884-900 - \\ Jaboticabal, SP. \\ ${ }^{3}$ Depto. de Ciências Exatas, FCAV/UNESP. \\ ${ }^{4}$ Bolsista FAPESP. \\ ${ }^{5}$ Bolsista CNPq. \\ ${ }^{*}$ Autor correspondente <rdvieira@fcav.unesp.br>
}

RESUMO: A temperatura interna das câmaras e o teor de água das sementes podem influenciar os resultados obtidos no teste de envelhecimento acelerado (EA) e, consequentemente, sua interpretação. $O$ trabalho foi desenvolvido com o objetivo de avaliar o efeito de diferentes teores iniciais de água de sementes de feijão nos resultados do teste de envelhecimento acelerado e as condições de temperatura e umidade relativa do ar no interior de duas câmaras de envelhecimento e das caixas plásticas (câmaras internas), durante a condução do teste. Utilizaram-se amostras de quatro lotes de sementes de feijão, cujo teor de água inicial foi ajustado para 8, 10 e 12\%. As sementes foram submetidas ao envelhecimento em câmaras "jaquetada" de água e de germinação tipo BOD. Durante a realização do teste, foram monitorados, com um registrador de dados, os valores da temperatura e da umidade relativa do ar no interior das câmaras e das caixas plásticas, avaliados a cada 10 minutos, por sensores de cobre-constantan com bulbos seco e úmido, instalados no interior das mesmas. Verificou-se pequena variação de temperatura e umidade relativa do ar nas duas câmaras, sendo, porém, menor na "jaquetada". Em função da umidade relativa do ar na câmara "jaquetada" ter sido mais elevada, as sementes envelhecidas nessa câmara, apresentaram teor de água mais alto do que aquelas envelhecidas na BOD. Ambas as câmaras podem ser utilizadas para a realização do teste de envelhecimento acelerado de sementes de feijão.

Palavras-chave: Phaseolus vulgaris, teor de água inicial, vigor

\section{EFFECT OF DRY BEAN INITIAL SEED MOISTURE CONTENT AND THE CHAMBER ON THE ACCELERATED AGING TEST}

\begin{abstract}
Chamber temperature and seed moisture content can influence the accelerated aging test results and consequently their interpretation. This work was conducted to determine the effect of initial dry bean seed moisture content on the accelerated aging test results, and the conditions of temperature and relative humidity in the two aging chambers and the gerbox (inner chambers), during the test. Samples obtained from four seed lots of dry beans were adjusted to 8,10 and $12 \%$ initial seed moisture. The seed samples were aged using two chambers (water-jacketed chamber) and a germination chamber (BOD type). During the test, the conditions of the temperature and the relative humidity in the outer and inner chambers (gerbox) were measured using a datalogger. There was little variation in the temperature and relative humidity for both chambers, being however lower for the water-jacketed chamber. Due to the higher relative humidity in the water-jacketed chamber, the seed moisture content was higher when compared to the BOD chamber. Both chambers (water-jacketed and $\mathrm{BOD}$ ) can be used for the dry bean seed accelerated aging test.
\end{abstract}

Key words: Phaseolus vulgaris, initial seed moisture content, vigor

\section{INTRODUÇÃO}

Avaliar a qualidade de um lote de sementes em termos de estimar com que sucesso ele estabelecerá uma população adequada de plântulas em campo, sob uma ampla faixa de condições ambientais, é de grande importância para atingir eficiência na agricultura moderna (Arthur \& Tonkin, 1991).

A tecnologia de sementes tem procurado aprimorar os procedimentos para avaliar a qualidade de sementes, com o objetivo de obter resultados que expressem o desempenho potencial de um lote, destacando-se em particular, os estudos relativos aos testes de vigor (Vieira, 1994)

O objetivo básico dos testes de vigor é a identificação de diferenças importantes na qualidade fisiológica entre lotes de sementes comercializáveis, principalmente, daqueles com poder germinativo semelhante. Daí, a importância da disponibilidade de testes que procuram avaliar o estado atual das sementes, através da determinação de parâmetros associados ao vigor. Existem, também, testes dirigidos

${ }^{1}$ Parte do trabalho de Graduação do primeiro autor apresentado à FCAV/UNESP - Jaboticabal, SP. 
à avaliação do comportamento das sementes quando submetidas a condições específicas de ambiente, geralmente estresses. Entretanto, têm sido relatadas dificuldades expressivas quanto à utilização extensiva dos testes de vigor, particularmente, no que diz respeito à falta de padronização de procedimentos, à interpretação subjetiva de resultados e às discrepâncias nos resultados obtidos entre e dentro de laboratórios.

Dentre os testes de vigor, destaca-se o de envelhecimento acelerado, que como vários outros, apresenta uma série de fatores que podem afetar e/ou dificultar a interpretação dos resultados obtidos (Tomes et al., 1988; Marcos Filho, 1999). Este teste tem sido indicado para várias espécies de sementes (Marcos Filho, 1999), porém, tem também, sido difícil a obtenção de resultados uniformes entre e dentro de laboratórios.

No Brasil, um dos problemas enfrentados pelos laboratórios diz respeito ao uso de equipamentos não confiáveis tecnicamente. Grandes variações de temperatura durante o processo de envelhecimento podem ocorrer e dependem do equipamento em uso (Bittencourt et al., 1995).

\section{MATERIAL E MÉTODOS}

Foram utilizadas amostras de três lotes de sementes de feijão das variedades IAC-CARIOCA (2 lotes) e Carioca Cobec, apresentando diferentes porcentagens de germinação e teores iniciais de água (TABELA 1).

As amostras de sementes de cada lote foram subdivididas em três subamostras e seus teores de água ajustados, respectivamente, para $8 \%, 10 \%$ e $12 \%$, através do cálculo da perda de água durante secagem $a \pm 38^{\circ} \mathrm{C}$ em câmara com circulação forçada de ar e/ou umedecimento das sementes, com base na pesagem das mesmas. Para tanto, utilizou-se a fórmula empregada por Martins et al. (1999): Px = Po (100-Uo/ 100-Ux); onde, Px é o peso da amostra de sementes a ser obtido; Po é o peso inicial da amostra; Uo é o teor inicial de água das sementes e Ux é o teor de água desejado nas sementes.

TABELA 1 - Teor de água e germinação de sementes de feijão, obtidos na avaliação inicial da qualidade das sementes.

\begin{tabular}{lcc}
\hline Lote & Teor de água & Germinação \\
\hline & ------ \\
\hline IAC-Carioca1 (lote 1) & $12,2 \mathrm{~A}$ & $90 \mathrm{~A}$ \\
IAC-Carioca1 (lote 2) & $12,4 \mathrm{~A}$ & $81 \mathrm{~B}$ \\
Carioca-Cobec & $8,4 \mathrm{~B}$ & $75 \mathrm{C}$ \\
\hline Média & 11,0 & 82,0 \\
\hline C.V. $(\%)$ & 1,7 & 2,3 \\
DMS $(0,05)$ & 0,4 & 3,7 \\
\hline
\end{tabular}

Médias seguidas pela mesma letra nas colunas não diferem pelo teste de Tukey a $5 \%$.
Após o ajuste dos teores de água, as sementes de cada subamostra foram submetidas a testes de envelhecimento acelerado (EA) conduzidos em duas câmaras de envelhecimento: a câmara "jaquetada" de água (VWR Scientific, modelo 3015) e a câmara de germinação tipo BOD (FANEM, modelo 347 CDG).

Imediatamente após o envelhecimento acelerado, determinou-se o teor de água das sementes pelo método da estufa a $105 \pm 3^{\circ} \mathrm{C}$ por 24 horas, seguindo-se os critérios descritos nas Regras para Análise de Sementes (Brasil, 1992).

Os testes de envelhecimento acelerado foram conduzidos pelo método da caixa plástica (câmara interna) de acordo com as recomendações disponíveis na literatura (Association of Official Seed Analysts, 1983; Hampton \& Tekrony, 1995; Marcos Filho, 1994), empregando-se as condições de $41^{\circ} \mathrm{C}$ por 72 horas (Hampton \& Tekrony, 1995). Após o envelhecimento, as sementes foram submetidas ao teste padrão de germinação (quatro repetições de 50 sementes) em substrato de papel toalha (Germitest, tipo CEL 065), de acordo com as Regras para Análise de Sementes (Brasil, 1992), computando-se a porcentagem de plântulas normais no quinto dia após a instalação do teste.

Durante o envelhecimento acelerado das sementes, foram monitoradas a temperatura e a umidade relativa do ar no interior das câmaras de envelhecimento e das caixas plásticas (câmaras internas), utilizando-se termopares de cobre-constantan com sensores de bulbo seco e úmido, cujos dados foram avaliados a cada 10 minutos e armazenados em um registrador de dados (21 XL Campbell Scientific).

$\mathrm{O}$ delineamento experimental utilizado foi em blocos casualizados com os tratamentos dispostos em esquema fatorial $2 \times 3$ (duas câmaras de envelhecimento $x$ três teores iniciais de água das sementes) com quatro repetições. Foi efetuada a análise da variância dos dados, aplicando-se o teste $\mathrm{F}$ e comparando-se as médias dos tratamentos pelo teste de Tukey a $5 \%$.

\section{RESULTADOS E DISCUSSÃO}

Os resultados de temperaturas máxima e mínima, no interior da câmara "jaquetada" de água e da BOD são apresentados na Figura 1. Observou-se que a maior variação entre as temperaturas máxima e mínima, atingindo valores próximos a $2^{\circ} \mathrm{C}$, ocorreu no período de 0 a 6 horas de envelhecimento das sementes na câmara "jaquetada" de água (1A). As avaliações das temperaturas, feitas no período de 6 a 72 horas, indicaram variações entre 0,2 e $0,3^{\circ} \mathrm{C}$. Isso ocorreu independentemente do teor de água inicial das sementes.

É necessário ressaltar, porém, que de acordo com Vieira (1999), a câmara de envelhecimento tipo "jaquetada" de água apresenta baixa variação da temperatura interna, quando a mesma é mantida em um 
ambiente onde a temperatura não apresenta grande variação entre a máxima e mínima durante o dia. Logo, ela exige um ambiente climatizado.

Para o envelhecimento realizado na câmara BOD (1B), verificaram-se, de modo geral, resultados semelhantes de temperatura média entre os períodos avaliados e também, de variação entre a máxima e mínima. O fato que mais chamou a atenção diz respeito à variação das temperaturas nas primeiras seis horas de envelhecimento. Ao contrário do que havia sido constatado com a câmara "jaquetada", a variação entre as temperaturas máxima e mínima na $B O D$, seguiu o mesmo padrão de variação em todo o período de envelhecimento ( 0 a 72 horas). Neste caso, a variação da temperatura interna da câmara de envelhecimento tipo BOD, apresentou valores mais altos, atingindo até $0,5^{\circ} \mathrm{C}$

A variação mais acentuada de temperatura, verificada em ambas as câmaras, nas primeiras seis horas de envelhecimento em relação aos períodos subsequentes, só foi detectada nos registros das avaliações feitas pelos termopares. Os valores mostrados nos termostatos das câmaras coincidiam com o valor de calibração de $42^{\circ} \mathrm{C}$.

Estes dados demostram que as variações de temperatura no interior da câmara, só pôde ser detectada, com precisão, quando as leituras foram realizadas em diferentes posições da mesma (dentro da área ocupada pelas caixas plásticas), concordando, portanto, com as observações feitas por Tomes et al. (1988) de que o monitoramento da temperatura da câmara de envelhecimento com base apenas nos valores do termostato do aparelho, não é totalmente confiável.

Na Figura 2, são apresentados os dados de temperaturas máxima e mínima no interior das caixas plásticas (câmaras internas) durante o processo de envelhecimento acelerado na câmara "jaquetada" de água (2A) e na câmara BOD (2B). Para a câmara "jaquetada", nos três teores iniciais de água avaliados, verificou-se que até se completarem seis horas de envelhecimento, houve uma variação de cerca de $4^{\circ} \mathrm{C}$ entre os valores de temperaturas máxima e mínima. Entre 6 e 72 horas de envelhecimento, a variação da temperatura foi menor, não superando $0,2^{\circ} \mathrm{C}(2 \mathrm{~A})$. Para a câmara tipo $\mathrm{BOD}(2 \mathrm{~B})$ a maior variação entre as temperaturas máxima e mínima também ocorreu no período de 0 a 6 horas de envelhecimento, atingindo, porém, valores mais baixos do que a "jaquetada" de água, próximos a $1^{\circ} \mathrm{C}$. As variações entre temperaturas máximas e mínimas nos períodos subsequentes, de forma semelhante a câmara "jaquetada", não ultrapassaram $0,2^{\circ} \mathrm{C}$.

A maior variação de temperatura nas primeiras seis horas de avaliação, observadas no interior das caixas plásticas em relação ao ambiente interno de ambas as câmaras decorreu, provavelmente, do tempo
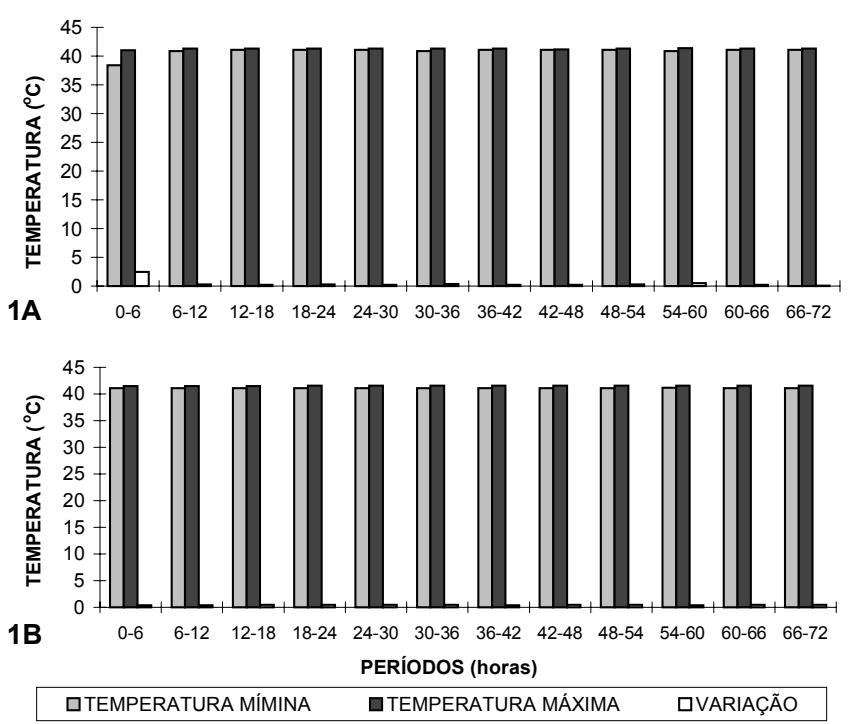

Figura 1 - Variação das temperaturas máxima e mínima, no interior da câmara "jaquetada" de água (1A) e da câmara tipo BOD (1B), durante o envelhecimento acelerado de sementes de feijão.
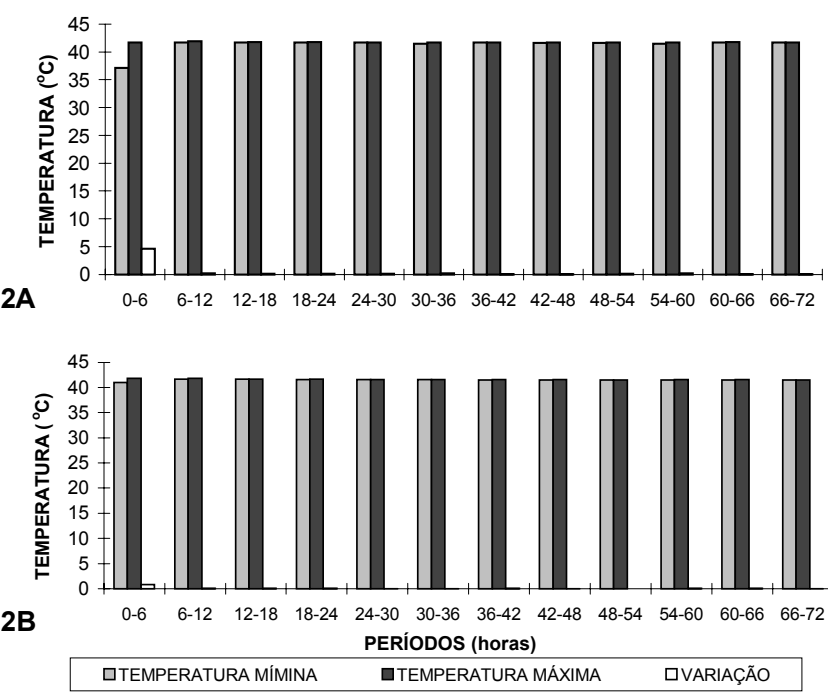

Figura 2 - Variação das temperaturas máxima e mínima, no interior das caixas plásticas (câmaras internas), na câmara "jaquetada" de água (2A) e na câmara tipo BOD (2B), durante o envelhecimento acelerado de sementes de feijão.

necessário para estabilizar as condições de temperatura e umidade relativa no interior das caixas.

Fato interessante, entretanto, é que nos períodos subsequentes ( 6 a 72 horas), em ambas as câmaras, o ambiente de acondicionamento das sementes (interior das caixas plásticas), cujas condições estão mais diretamente relacionadas ao processo de envelhecimento das mesmas, a variação de temperatura foi sempre inferior a $0,3^{\circ} \mathrm{C}$. Este limite de variação de temperatura, segundo Marcos filho (1999), é um dos principais fatores a ser considerado na precisão dos resultados do teste de envelhecimento acelerado. 
$\mathrm{Na}$ Figura 3 são apresentados os dados referentes à umidade relativa do ar (UR) no interior das caixas plásticas (câmaras internas). Em ambas as câmaras, a UR do ar foi sempre superior a $90 \%$ no interior das caixas plásticas durante o envelhecimento. Os menores valores de UR do ar obtidos, ocorreram sempre nas primeiras horas de envelhecimento $(0-12$ horas), independente do teor inicial de água das sementes.

A UR do ar dentro da câmara e no interior das caixas plásticas (câmaras internas) foi sempre maior na câmara "jaquetada" de água, quando comparada à BOD (Figura 3). Entretanto, valores superiores à $90 \%$ de umidade relativa do ar dentro da caixa plástica, conforme observado nas duas câmaras de envelhecimento avaliadas, são considerados adequados para a realização do teste de envelhecimento acelerado.

Após o ajuste dos teores de água das sementes $(8,10$ e $12 \%)$, os valores obtidos não diferiram estatisticamente entre si, indicando que o método usado para obter os teores de água desejados, foi eficiente. Quando ajustado para 8\%, obtiveram-se para os quatro lotes valores entre 7,8 e $8,2 \%$; para $10 \%$, entre 9,9 e $10,2 \%$ e para $12 \%$, entre 12,1 e $12,2 \%$.

Na câmara "jaquetada" de água as sementes dos três lotes avaliados atingiram, após o envelhecimento acelerado, teores de água mais elevados que na BOD (TABELA 2). Considerando-se que o teor de água das sementes, é acima de tudo, função da umidade relativa do ar (Carvalho \& Nakagawa, 2000), estes resultados estão de acordo com os dados da Figura 3, que indicam a ocorrência de valores mais altos de umidade relativa do ar na câmara "jaquetada" do que na BOD.

Na câmara "jaquetada" de água, as sementes do IAC-Carioca (lote 1) e do Carioca-Cobec, com teor inicial de água de 8,10 e $12 \%$, atingiram após 0 envelhecimento acelerado, valores crescentes de umidade. Resultados semelhantes, na BOD, só ocorreram para as sementes do Carioca-Cobec. Nas demais situações, não houve consistência dos resultados, verificando-se, por exemplo, que as sementes do IAC-Carioca (lote 2) com teor mais baixo de água $(8 \%)$, foi as que atingiram umidade mais elevada após o envelhecimento (TABELA 2).

Independente da câmara, a germinação após o envelhecimento acelerado, dos lotes de sementes com teor de água inicial de 8 e 10\%, não sofreu efeito significativo dos teores iniciais de água (TABELAS 3). A germinação das sementes da variedade IAC-Carioca (lote 2), envelhecidas com $12 \%$ de água, diferiu significativamente daquelas que apresentavam teor inicial de água de $8 \%$.

Estes resultados indicam que diferenças superiores a dois pontos percentuais de teor inicial de água entre amostras de sementes de feijão, podem comprometer os resultados do teste de envelhecimento acelerado, concordando com os relatos de Marcos Filho (1999). Segundo este pesquisador, diferenças acentuadas de teores iniciais de água das sementes condicionam variação na velocidade de umedecimento durante o envelhecimento e, consequentemente, diferenças na intensidade de deterioração.

Observa-se, ainda na TABELA 3 , que os resultados do teste de envelhecimento acelerado não foram alterados em função da câmara utilizada. Os valores de germinação foram estatisticamente semelhantes, dentro de cada um dos lotes.

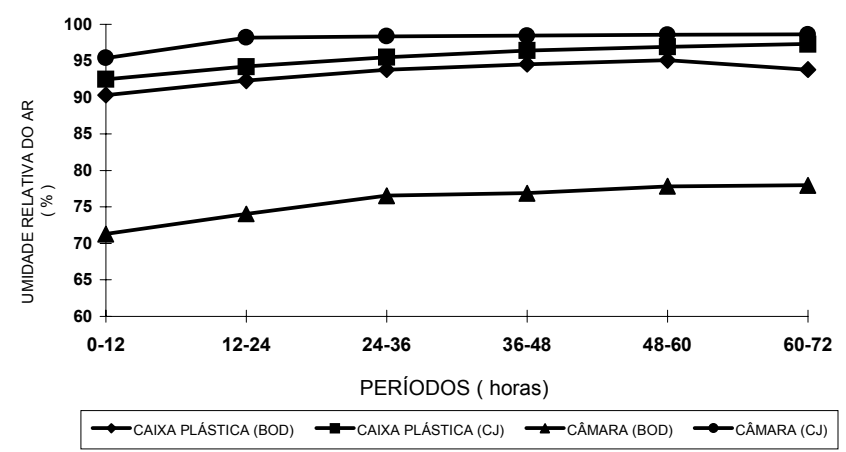

Figura 3 - Variação da umidade relativa do ar no interior das câmaras e das caixas plásticas (câmaras internas), durante o envelhecimento acelerado de sementes defeijão.

TABELA 2 - Teor de água após o envelhecimento acelerado, atingido por sementes de feijão com três teores iniciais de água, em duas câmaras de envelhecimento: BOD e "jaquetada" de água (CJ).

\begin{tabular}{|c|c|c|}
\hline Teor inicial de água & BOD & CJ \\
\hline \multicolumn{3}{|c|}{ AC-Carioca (lote 1) } \\
\hline \multicolumn{3}{|c|}{ 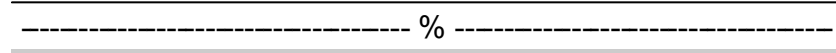 } \\
\hline 8 & $28,7 \mathrm{Bb}$ & $32,1 \mathrm{Ca}$ \\
\hline 10 & $29,7 \mathrm{Ab}$ & $32,9 \mathrm{Ba}$ \\
\hline 12 & $29,5 \mathrm{Ab}$ & $33,9 \mathrm{Aa}$ \\
\hline Média & $29,3 \mathrm{~b}$ & 33,0 a \\
\hline $\mathrm{CV}(\%)=0,7 \quad \mathrm{DMS}($ & lunas) $=0$ & DMS (linhas) $=0,3$ \\
\hline \multicolumn{3}{|c|}{ IAC-Carioca (lote 2) } \\
\hline \multicolumn{3}{|c|}{ - } \\
\hline 8 & $31,0 \mathrm{Ab}$ & $32,6 \mathrm{Ba}$ \\
\hline 10 & $30,7 \mathrm{Bb}$ & $32,2 \mathrm{Ca}$ \\
\hline 12 & $28,3 \mathrm{Cb}$ & $33,7 \mathrm{Aa}$ \\
\hline Média & $30,0 \mathrm{~b}$ & $32,9 \mathrm{a}$ \\
\hline $\mathrm{CV}(\%)=0,4$ DMS $(\mathrm{c}$ & unas) $=0$ & DMS (linhas) $=0,2$ \\
\hline \multicolumn{3}{|c|}{$\begin{array}{c}\text { Carioca-Cobec } \\
\end{array}$} \\
\hline \multicolumn{3}{|c|}{ - } \\
\hline 8 & $27,3 \mathrm{C} \mathrm{b}$ & $31,8 \mathrm{C} \mathrm{a}$ \\
\hline 10 & $29,6 \mathrm{~B} \mathrm{~b}$ & $32,7 \mathrm{~B}$ a \\
\hline 12 & $31,4 \mathrm{~A} \mathrm{~b}$ & $33,3 \mathrm{~A}$ a \\
\hline Média & $29,4 \mathrm{~b}$ & $32,6 \mathrm{a}$ \\
\hline
\end{tabular}

Médias seguidas pela mesma letra maiúscula nas colunas e minúsculas nas linhas não diferem pelo teste de Tukey a $5 \%$. 
TABELA 3 - Germinação após o envelhecimento acelerado, de sementes de feijão com três teores iniciais de água, em duas câmaras de envelhecimento: BOD e "jaquetada" de água (CJ).

\begin{tabular}{|c|c|c|c|}
\hline Teor inicial de água & BOD & CJ & Média \\
\hline \multicolumn{4}{|c|}{ AC-Carioca (lote 1) } \\
\hline \multicolumn{4}{|c|}{ 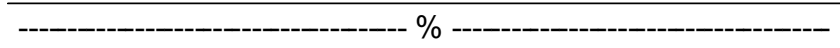 } \\
\hline 8 & 84 & 85 & $84,5 \mathrm{~A}$ \\
\hline 10 & 84 & 81 & $82,5 \mathrm{~A}$ \\
\hline 12 & 84 & 80 & $82,0 \mathrm{~A}$ \\
\hline Média & $84,0 \mathrm{a}$ & $82,0 \mathrm{a}$ & \\
\hline $\mathrm{CV}(\%)=4,6$ DMS $(\mathrm{c}$ & nas) $=4,9$ & DMS (lint & s) $=3,3$ \\
\hline \multicolumn{4}{|c|}{ AC-Carioca (lote 2) } \\
\hline \multicolumn{4}{|c|}{ 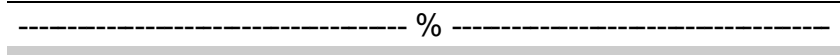 } \\
\hline 8 & 66 & 64 & $65,3 \mathrm{~A}$ \\
\hline 10 & 66 & 62 & $64,3 \mathrm{AB}$ \\
\hline 12 & 54 & 58 & $56,3 \mathrm{~B}$ \\
\hline Média & 62,0 a & $61,3 \mathrm{a}$ & \\
\hline $\mathrm{CV}(\%)=9,9$ DMS $(\mathrm{c}$ & nas ) $=8,8$ & DMS (linh & s) $=5,9$ \\
\hline \multicolumn{4}{|c|}{$\begin{array}{l}\text { Carioca-Cobec } \\
\end{array}$} \\
\hline \multicolumn{4}{|c|}{ 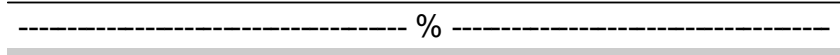 } \\
\hline 8 & 72 & 73 & $72,5 \mathrm{~A}$ \\
\hline 10 & 68 & 65 & $66,5 \mathrm{~A}$ \\
\hline 12 & 69 & 63 & $66,0 \mathrm{~A}$ \\
\hline Média & 69,7 a & $67,0 \mathrm{a}$ & \\
\hline $\mathrm{CV}(\%)=7,9$ DMS $(\mathrm{c}$ & nas ) $=7,1$ & DMS (linh & s) $=4,8$ \\
\hline
\end{tabular}

Médias seguidas pela mesma letra maiúscula nas colunas e minúsculas nas linhas não diferem pelo teste de Tukey a $5 \%$.

\section{CONCLUSÕES}

Ambas as câmaras avaliadas ("jaquetada" de água e BOD) podem ser usadas para a condução do teste de envelhecimento acelerado para sementes de feijão. O fator importante é que para a classificação dos lotes de sementes quanto ao nível de vigor, devem-se comparar os resultados de testes de envelhecimento acelerado realizados no mesmo tipo de câmara.

\section{REFERÊNCIAS BIBLIOGRÁFICAS}

ARTHUR, T.J.; TONKIN, J.H.B. Testando o vigor da semente. Informativo ABRATES, v.1, p.38-41, 1991.

ASSOCIATION OF OFFICIAL SEED ANALYSTS. Seed vigour testing handbook. East Lansing: AOSA, 1983. 93p. (Contribution, 32).

BITTENCOURT, S.R.M.; VIEIRA, R.D.; BARRETO, M.; VOLPE, C.A. Comparação de dois tipos de germinadores como câmaras de envelhecimento acelerado. Revista Brasileira de Sementes, v.17, p.160-164, 1995.

BRASIL. Ministério da Agricultura e Reforma Agrária. Regras para análise de sementes. Brasília: SNDA, DNDV, CLV, 1992. 365p.

CARVALHO, N.M.; NAKAGAWA, J. Sementes: ciência, tecnologia e produção. Jaboticabal: FUNEP, 2000. 588p.

HAMPTON, J.G.; TEKRONY, D.M. Handbook of vigor test methods. Zürich: ISTA, 1995. 117p.

MARCOS FILHO, J. Teste de envelhecimento acelerado. In: VIEIRA, R.D.; CARVALHO, N.M. (Ed.) Testes de vigor em sementes. Jaboticabal: FUNEP, 1994. p.133-149.

MARCOS FILHO, J. Teste de envelhecimento acelerado. In: KRZYZANOWSKI, F.C.; VIEIRA, R.D.; FRANÇA NETO, J.B. (Ed.) Vigor de sementes: conceitos e testes. Londrina: ABRATES, 1999. cap.3, p.1-24.

MARTINS, C.C.; NAKAGAWA, J.; BOVI, M.L.A.; STANGUERLIM, $\mathrm{H}$. Teores de água crítico e letal para sementes de açaí (Euterpe oleracea Mart.-PALMAE). Revista Brasileira de Sementes, v.21, p.125-132, 1999.

TOMES, L.J.; TEKRONY, D.M.; EGLI, D.B. Factors influencing the tray accelerated ageing test for soybean seed. Journal of Seed Technology, v.12, p.24-36, 1988.

VIEIRA, R.D. Testes de vigor e suas possibilidades de uso. In: VIEIRA, R.D.; CARVALHO, N.M. (Ed.) Testes de vigor em sementes. Jaboticabal: FUNEP, 1994. p.31-47.

VIEIRA, R.D. Testes de vigor utilizados para sementes de soja no Brasil e na atualidade. In: CONGRESSO BRASILEIRO DE SOJA, 1., Londrina, 1999. Anais. Londrina: Embrapa Soja, 1999. p.227-232 (Documentos, 124).

$\overline{\text { Recebido em } 07.08 .00}$ 